\title{
Experiencias psicóticas atenuadas y consumo de sustancias en universitarios
}

\section{Psychotic-like Experiences and Substance Use in College Students}

\author{
Eduardo Fonseca-Pedrero********, Javier Ortuño-Sierra*****, Mercedes Paino**,*** \\ Y José MUÑIZ***,****. \\ * Departamento de Ciencias de la Educación. Universidad de La Rioja, España. ** Programa de Prevención en Psicosis (P3), \\ España. *** Departamento de Psicología. Universidad de Oviedo, España. **** Centro de Investigación Biomédica en Red de \\ Salud Mental (CIBERSAM). ***** Universidad Loyola Andalucía, España.
}

\section{Resumen}

Los trastornos del espectro esquizofrénico, así como las experiencias psicóticas, se han asociado con un mayor consumo de sustancias. El objetivo de este trabajo fue analizar la relación entre las experiencias psicóticas atenuadas y el consumo de sustancias en adultos jóvenes. La muestra la formaron un total de 660 participantes universitarios (M $=20,3$ años; DT $=2,6$ ). Los resultados mostraron que un $96 \%$ de la muestra informó de alguna experiencia de ideación delirante, mientras que el 20,3\% informó de, al menos, una experiencia atenuada de tipo cognitivo-perceptual. El $41,1 \%$ de la muestra refirió algún consumo de sustancias, encontrándose diferencias en función del género. Los participantes consumidores informaron de un mayor número de experiencias psicóticas, sobre todo de tipo positivo. Asimismo, el consumo de alcohol predijo, en la mayoría de los casos, las puntuaciones extremas en las medidas de ideación delirante y experiencias pseudopsicóticas. La asociación entre estas dos variables parece mostrar un patrón diferenciado, encontrándose el consumo de sustancias más relacionado con las experiencias pseudo-psicóticas de tipo cognitivoperceptual. Estos hallazgos parecen apoyar los modelos dimensionales del fenotipo psicótico y permiten mejorar la comprensión de la relación entre las experiencias psicóticas atenuadas y el consumo de sustancias en adultos jóvenes. Futuros estudios deberían seguir analizando el papel de los factores de riesgo a los trastornos psicóticos, así como incorporar modelos de interacción gen $\mathrm{x}$ ambiente.

Palabras clave: Consumo de sustancias; Adicción; Psicosis; Esquizotipia; Cannabis; Experiencias psicóticas atenuadas.

\begin{abstract}
Psychotic disorders, as well as psychotic-like experiences and substance use, have been found to be associated. The main goal of the present study was to analyse the relationship between psychoticlike experiences and substance use in college students. The sample comprised a total of 660 participants $(\mathrm{M}=20.3$ years, $\mathrm{SD}=2.6)$. The results showed that $96 \%$ of the sample reported some delusional experience, while $20.3 \%$ reported at least one positive psychotic-like experience. Some substance use was reported by $41.1 \%$ of the sample, differing in terms of gender. Substance users reported more psychoticlike experiences than non-users, especially in the positive dimension. Also, alcohol consumption predicted in most cases extreme scores on measures of delusional ideation and psychotic experiences. The association between these two variables showed a differentiated pattern, with a stronger relationship between substance use and cognitive-perceptual psychotic-like experiences. To some extent, these findings support the dimensional models of the psychosis phenotype and contribute a better understanding of the links between psychoticlike experiences and substance use in young adults. Future studies should further explore the role of different risk factors for psychotic disorders and include models of the gene-environment interaction. Keywords: Substance use; Addiction; Psychosis; Schizotypy; Cannabis; Psychotic-like experiences.
\end{abstract}


$\mathrm{E}$ l abuso y consumo de sustancias es frecuente en los pacientes con trastornos del espectro psicótico (Buckley, Miller, Lehrer, y Castle, 2009). El uso y abuso de sustancias ha sido asociado con un peor curso clínico, un inicio más temprano, una mayor duración de la psicosis sin tratar, exacerbación de la sintomatología, así como un mayor número de recaídas y hospitalizaciones y una peor adherencia al tratamiento, entre otros aspectos (Broussard et al., 2013; González-Pinto et al., 2011; Henquet et al., 2010; Lambert et al., 2005; Stefanis et al., 2014; Wade et al., 2006; Wisdom, Manuel, y Drake, 2011; Zammit et al., 2008). Además, el consumo previo de determinadas sustancias, como por ejemplo el cannabis, parece incrementar el riesgo posterior de desarrollar psicosis, así como las tasas de síntomas psicóticos subclínicos y experiencias psicóticas atenuadas, tanto en población general (Henquet, Murray, Linszen, y Van Os, 2005; Kuepper et al., 2011; McGrath et al., 2010; Moore et al., 2007), como en familiares de pacientes con psicosis (McGuire et al., 1995). Por ejemplo, estudios epidemiológicos han encontrado que el inicio en el uso de cannabis en la adolescencia incrementa la probabilidad de experimentar síntomas y trastornos del espectro psicótico en la etapa adulta (Arseneault et al., 2002) y favorece la persistencia de experiencias psicóticas atenuadas (Mackie et al., 2013). Similares resultados se hallan cuando se analiza longitudinalmente a participantes de alto riesgo clínico (estados mentales de alto riesgo), si bien por el momento no se dispone de suficiente evidencia que permita asociar el uso de sustancias con una mayor probabilidad de transición a la psicosis (Addington et al., 2014).

El abordaje dimensional del fenotipo psicótico sugiere que las experiencias psicóticas (p.ej., ideación delirante, pensamiento mágico) se distribuyen dentro de un continuo de gravedad, situándose en su parte más extrema el cuadro psicótico (van Os, Linscott, Myin-Germeys, Delespaul, y Krabbendam, 2009). Tales experiencias psicóticas, aunque de forma atenuada, se pueden encontrar distribuidas normalmente en la población general, sin estar necesariamente asociadas a malestar, discapacidad y necesidad de tratamiento (Linscott y van Os, 2013; McGrath et al., 2015). A este conjunto de experiencias atenuadas, cuando persisten en el tiempo y se acompañan de cierto malestar, preocupación y búsqueda de ayuda, se les suele denominar síntomas psicóticos atenuados (Fonseca-Pedrero, Paino, y Fraguas, 2013; Yung et al., 2007). Este tipo de experiencias atenuadas son consideradas como un marcador fenotípico de vulnerabilidad para los trastornos del espectro psicótico, en general, y para la esquizofrenia en particular (Debbané et al., 2015; Linscott y van Os, 2013). En este sentido, es posible que la identificación de este conjunto de experiencias subclínicas permita la detección e identificación temprana de participantes vulnerables a un trastorno mental grave, como la psicosis. Asimismo, este tipo de experiencias pseudo-psicóticas se podrían utilizar en combinación con otros marcadores de riesgo (proximales o distales), por ejemplo, el consumo o abuso de sustancias, el trauma o la ur- banicidad (van Os, Kenis, y Rutten, 2010), con la finalidad de examinar posibles mecanismos etiológicos subyacentes, así como mejorar las estrategias y programas de detección precoz y prevención temprana. El posible efecto del consumo de sustancias en individuos de riesgo a la psicosis podría estar modulado por diferentes factores como, por ejemplo, la edad de primer consumo, el patrón y la frecuencia de uso de sustancias o la vulnerabilidad preexistente (Casadio, Fernandes, Murray, y Di Forti, 2011; Valmaggia et al., 2014).

Estudios previos han asociado las experiencias psicóticas atenuadas con el consumo y abuso de sustancias, especialmente de cannabis (Barkus, Stirling, Hopkins, y Lewis, 2006; Mackie et al., 2013; Saha et al., 2011; Schubart et al., 2011; van Os et al., 2002). Los resultados parecen indicar que la asociación es más probable y aumenta entre aquellos participantes que tienen cierta predisposición latente o informan de historia familiar de psicosis (Henquet, Krabbendam, et al., 2005; Stowkowy y Addington, 2013; van Os et al., 2002). En un estudio longitudinal, van Os et al. (2002) encontraron que el consumo de cannabis predijo la presencia de síntomas psicóticos al cabo de tres años, así como su nivel de gravedad y la necesidad de tratamiento. En otro estudio epidemiológico realizado en Australia, Saha et al. (2011) hallaron que las experiencias delirantes se asociaban con un mayor consumo de tabaco, cannabis y alcohol. Estos trabajos analizan preferentemente el rol de las experiencias psicóticas atenuadas de tipo positivo (p.ej., la ideación delirante o las experiencias alucinatorias), si bien el efecto de las experiencias pseudo-psicóticas de tipo negativo (p.ej., afecto aplanado o déficit para experimentar placer) no se encuentra analizado en profundidad (Schubart et al., 2011).

Resultados convergentes han sido encontrados cuando se examina la relación entre los rasgos esquizotípicos y el consumo de sustancias (Barkus et al., 2006; Davis, Compton, Wang, Levin, y Blanco, 2013; Fumero, Santamaría, y Navarrete, 2009; Najolia, Buckner, y Cohen, 2012; Nunn, Rizza, y Peters, 2001). Los rasgos esquizotípicos se encuentran íntimamente relacionados con las experiencias psicóticas atenuadas siendo, en ocasiones, utilizados como términos intercambiables (Kwapil y Barrantes-Vidal, 2015). Los dos constructos se distribuyen normalmente en la población general dentro de un continuo psicótico de gravedad, si bien los rasgos esquizotípicos aglutinan un mayor conjunto de manifestaciones psicóticas atenuadas (p.ej., dimensiones positiva, negativa y desorganizada) y son de carácter más bien estable (rasgo). Por su parte, las experiencias pseudo-psicóticas se suelen referir, en mayor medida, a manifestaciones de tipo positivo o "florido" y son de carácter más bien transitorio (estado). $\mathrm{Al}$ igual que los estudios realizados con experiencias psicóticas atenuadas, los individuos con puntuaciones elevadas en medidas de esquizotipia, seguidos longitudinalmente, tienen mayor probabilidad de desarrollar trastornos por abuso de sustancias (Kwapil, 1996). Asimismo, estudios transversales han encontrado que los rasgos esquizotípicos son predicto- 
res del consumo de alcohol y tabaco en universitarios españoles (Fumero et al., 2009). En este sentido, diversos estudios indican que los consumidores de cannabis y/o alcohol presentan menores puntuaciones en la dimensión negativa de la esquizotipia en comparación con los no consumidores (Nunn et al., 2001; Skosnik, Park, Dobbs, y Gardner, 2008), si bien otros trabajos no encuentran tal asociación (Barkus et al., 2006; Dumas et al., 2002; Fridberg, Vollmer, O’Donnell, y Skosnik, 2011; Schiffman, Nakamura, Earleywine, y LaBrie, 2005), o incluso revelan una asociación positiva (Bailey y Swallow, 2004; Davis et al., 2013).

Como se puede observar, los trabajos que analizan la asociación entre las experiencias psicóticas atenuadas y el consumo de sustancias en muestras no clínicas de adultos jóvenes son inconsistentes. Más aún, el papel de las experiencias pseuso-psicóticas de tipo negativo no han sido aún objeto de estudio en profundidad. Por ello, resulta necesario llevar a cabo nuevas investigaciones que permitan conocer y ahondar en la relación entre este tipo de experiencias atenuadas y el consumo de sustancias, en una etapa del desarrollo de especial vulnerabilidad para la psicosis como es la temprana adultez. Dentro de este contexto de investigación, el principal propósito de este trabajo fue examinar la relación entre las experiencias psicóticas atenuadas y el consumo de sustancias en una muestra de adultos jóvenes universitarios. Se hipotetiza que el porcentaje de personas que informen de consumo de sustancias y de experiencias psicóticas atenuadas será elevado. Asimismo, en función de los trabajos previos, se hipotetiza que los consumidores de sustancias presentarán una mayor frecuencia de experiencias psicóticas atenuadas, tanto positivas como negativas, en comparación con los no consumidores, y que la asociación será más fuerte con las experiencias psicóticas de tipo cognitivo-perceptual.

\section{Método}

\section{Participantes}

Se ha utilizado una muestra incidental de estudiantes universitarios. La muestra, la conformaron un total de 660 participantes, 195 varones $(29,5 \%)$, pertenecientes a distintos estudios de la Universidad de Oviedo (Magisterio, Criminología, Psicología, Medicina, Logopedia, Informática, Economía y Fisioterapia). La media de edad de los participantes fue de 20,3 años $(D T=2,6)$, oscilando el rango de edad entre los 17 y los 30 años. La media del número de años de educación fue 16,8 $(D T=2,3)$. En relación con el estado civil, el $81,6 \%$ de la muestra estaba soltero, el 16,2\% casado, el $0,6 \%$ divorciado y el 1,7\% no informó de su estado. Respecto a su situación laboral, el 86,6\% de los participantes no trabajaba, el 12,6\% trabajaba y el 1,2\% no informó de su situación laboral. Los criterios de exclusión utilizados en el presente trabajo fueron: a) presencia de un trastorno mental grave (p.ej., psicosis, trastorno bipolar); y b) presencia de un trastorno neurológico.

\section{Instrumentos}

Cuestionario de consumo de sustancias. Para la evaluación del consumo de sustancias se formularon una serie de preguntas ad hoc referidas al consumo de alcohol y consumo de drogas, concretamente: cannabis, inhalantes, cocaína, drogas de diseño y heroína/morfína. Con relación al consumo de alcohol se recogió información sobre si el participante era no bebedor, bebedor o ex-bebedor. En el caso de los participantes que reflejaron ser bebedores, se preguntó sobre la cantidad de alcohol en unidades de gramos al día, atendiendo a cuatro categorías: hasta 10, de 11 a 20, de 21 a 50 y más de 51 . Asimismo, también se recogió información sobre otras sustancias como Cannabis/Marihuana/Hachís, Inhalantes, Cocaína, Drogas de diseño/Metanfetaminas/ Éxtasis/LSD y Heroína/Morfina. Para cada una de ellas se solicitó información de la edad de inicio del consumo en años, el tiempo de consumo en meses y los días de consumo en el último mes. Estas preguntas fueron formuladas atendiendo a trabajos previos (Dumas et al., 2002; Najolia et al., 2012) y escalas previamente validadas (Soto-Brandt et al., 2014).

Escala para la Evaluación Comunitaria de las Experiencias Psíquicas-42 (CAPE-42) (Stefanis et al., 2002). La CAPE se compone de 42 ítems que evalúan tres dimensiones de los síntomas psicóticos: Positiva (20 ítems), Negativa (14 ítems) y Depresiva (8 ítems). Cada pregunta se responde en un formato de respuesta tipo Likert de cuatro puntos que oscila desde Casi nunca (1) hasta Casi siempre (4). En el caso que el participante elija las opciones de respuesta Algunas veces, A menudo o Casi siempre, debe indicar el grado de malestar que le produce tal experiencia en una escala tipo Likert de cuatro puntos ( 0 = No me molesta nada; $3=$ Me molesta mucho $)$. En este trabajo se ha utilizado la versión adaptada al español y validada según los estándares internacionales (Muñiz, Elosua, y Hambleton, 2013). Las puntuaciones obtenidas en estudios previos presentan adecuados niveles de fiabilidad y diferentes evidencias de validez (Barragan, Laurens, Blas Navarro, y Obiols, 2011; Fonseca-Pedrero et al., 2011; Fonseca-Pedrero, Paino, Lemos-Giráldez, y Muñiz, 2012b).

Inventario de Ideas Delirantes de Peters et al.-21 (PDI-21) (Peters, Joseph, Day, y Garety, 2004). El PDI-21 es un autoinforme diseñado para la valoración de las experiencias delirantes y la propensión delirante. El PDI consta de un total de 21 ítems en formato de respuesta dicotómico Sí/ No. La puntuación total se obtiene sumando las respuestas positivas en cada uno de los ítems, por lo que la puntuación máxima que se puede alcanzar es de 21 puntos. Una mayor puntuación es indicativa de mayor sintomatología delirante o propensión delirante. Asimismo, cada uno de los ítems consta de tres subescalas que miden el grado de convicción, preocupación y malestar. En estas tres subescalas el sistema de puntuación es de tipo Likert de cinco categorías (1-5). En este trabajo se ha utilizado la versión española del PDI-21 que presenta adecuadas propiedades psicométricas (Fon- 
seca-Pedrero, Paino, Santarén-Rosell, Lemos-Giráldez, y Muñiz, 2012; López-Ilundain, Pérez-Nievas, Otero, y Mata, 2006).

Cuestionario de Ansiedad Estado-Rasgo (STAI) (Spielberger, Gorsuch, Lushene, Vagg, y Jacobs, 1983). El STAI es un autoinforme compuesto por 40 ítems diseñado para evaluar dos conceptos independientes de la ansiedad: Estado y Rasgo. Cada escala se conforma por un total de 20 ítems en un sistema de respuesta Likert de cuatro puntos según la intensidad $(0$ = Casi nunca $/$ nada; 3 = Mucho/casi siempre $)$. La puntuación total en cada una de las escalas oscila entre 0 y 60 puntos. En este trabajo se ha utilizado la adaptación española del STAI (Spielberger, Gorsuch, y Lushene, 2008). La versión española presenta niveles de consistencia interna que oscilan entre 0,84 y 0,93 , y las evidencias de validez han sido previamente analizadas (Fonseca-Pedrero, Paino, Lemos-Giráldez, y Muñiz, 2012a; Guillén-Riquelme y Buela-Casal, 2011).

\section{Procedimiento}

La administración de los instrumentos de medida se llevó a cabo de forma colectiva, en grupos de 10 a 45 estudiantes, durante el horario académico y en un aula acondicionada para este fin. El estudio fue presentado a los participantes como una investigación sobre las diversas características de la personalidad, asegurándoles la confidencialidad de sus respuestas, así como del carácter voluntario de su participación. La administración de los autoinformes se realizó en todo momento bajo la supervisión de un investigador. Todos los participantes dieron su consentimiento informado para la participación en la investigación. Este estudio se encuadra dentro de una línea de investigación relacionada con la detección temprana de trastornos psicológicos graves en jóvenes adultos. El estudio fue aprobado por el comité ético de la Universidad de Oviedo.

\section{Análisis de datos}

En primer lugar, se calcularon los estadísticos descriptivos para el PDI-21 y la CAPE-42. En segundo lugar, se analizó la prevalencia de experiencias psicóticas autoinformadas, así como para el consumo de sustancias. A continuación, en tercer lugar, se realizaron diferentes análisis multivariados de la covarianza (MANCOVA), controlando el efecto de las covariables género, ansiedad estado y ansiedad rasgo. Las variables dependientes fueron las puntuaciones del PDI-21 y la CAPE-42, mientras que los factores fijos fueron el consumo o no de sustancias, así como el poli-consumo global dicotomizado (no consumidores versus consumidores de alguna sustancia). No se consideró analizar ni el efecto de los inhalantes ni de la heroína por su baja prevalencia en la presente muestra. Para la estimación de las posibles diferencias estadísticamente significativas entre todas las variables se empleó el valor Lambda de Wilks. Para el cálculo del tamaño del efecto se utilizó el estadístico eta cuadrado parcial ( $\eta^{2}$ parcial).

En cuarto lugar, se llevaron a cabo diferentes análisis de regresión logística binaria de tipo jerárquico, para lo cual se dicotomizaron las puntuaciones del PDI-21 y la CAPE-42. Se seleccionó a los participantes con puntuaciones iguales o superiores al percentil 90, o a participantes con un percentil igual o inferior a 10. Se controló también el efecto del género, ansiedad estado y ansiedad rasgo. Las covariables fueron introducidas en el primer paso del modelo. Como variables fijas se seleccionaron los consumos de alcohol, cannabis, cocaína y drogas de diseño. El método para le estimación fue Adelante de Wald; se calcularon las odds ratio, así como su intervalo confidencial (95\%). La prueba de Hosmer Lemeshow se utilizó para evaluar la bondad del ajuste de un modelo de regresión logística binaría. Para el análisis de los datos se utilizó el paquete estadístico SPSS 15.0 (Statistical Package for the Social Sciences, 2006)

\section{Resultados}

\section{Estadísticos descriptivos y prevalencia de experien- cias psicóticas atenuadas y consumos}

Los estadísticos descriptivos referidos a las puntaciones de la CAPE-42 se pueden consultar en un trabajo previo (Fonseca-Pedrero, Paino, et al., 2012b). Los estadísticos del PDI-21 fueron: $M=4,30 ; S D=2,18$; rango 0-18; alfa de Cronbach = 0,91 .

El porcentaje de participantes que respondieron de forma afirmativa ( $A$ menudo o Casi siempre) en las opciones de respuesta de la dimensión positiva de la CAPE-42 osciló entre el $0,6 \%$ (ítem 24) y el 20,3\% (ítem 6), si bien es cierto que todos los porcentajes se acercan al $2 \%$. En el caso del PDI-21, el porcentaje de participantes que informó positivamente de alguna experiencia de tipo delirante fue del $96 \%$.

En relación al consumo de sustancias informado por los participantes se encontraron los siguientes resultados. El $32,4 \%(n=215)$ de los participantes se consideraron bebedores. El porcentaje por tipo de ingesta de consumo fue: $26,1 \%$ ( $n=172)$ menos de 10gr./día, 3,5\% $(n=23)$ de 11-20 gr./día, $1,8 \%(n=12)$ 21-50 gr./día, 0,3\% $(n=2)$ más de 51 gr./día y $0,9 \%(n=6)$ ex-bebedor. El $20,2 \%(n=133)$ de la muestra informó de consumo de cannabis, el $0,6 \%(n=4)$ de inhalantes, el 7,7\% $(n=51)$ de cocaína, el 2,6\% $(n=17)$ de drogas de diseño y el $0,2 \%(n=1)$ de heroína. En términos globales, un $41,1 \%$ de la muestra informó de algún consumo de sustancias, con la siguiente distribución: $0=58,9 \%(n=153), 1$ $=23,2 \%(n=153), 2=14,4 \% \quad(n=95), 3=2,3 \%(n=15), 4=$ $1,1 \%(n=7)$ y $5=0,2 \%(n=1)$. Si se considera el consumo, eliminando las sustancias con menor prevalencia (heroína e inhalantes), la distribución de número de sustancias fue: $0=$ $58,9 \%(n=389), 1=24,2 \%(n=153), 2=14,7 \%(n=97), 3=$ $2,3 \%(n=15)$, y $4=0,9 \%(n=6)$. Si únicamente se considera el consumo de las sustancias cannabis, cocaína y de diseño, el $75 \%$ de la muestra informó de ausencia de consumos.

Los patrones de consumo variaron de forma diferencial en función del sexo en el consumo de alcohol (dicotomizada consumo vs. no consumo) $(\chi 2(1)=30,78, p=0,001)$, canna- 
bis $(\chi 2(1)=21,31, p=0,001)$, inhalantes $(\chi 2(1)=9,59, p=$ $0,001)$ y drogas de diseño $(\chi 2(1)=4,58, p=0,032)$. En estos casos los varones refirieron mayor consumo de sustancias que las mujeres. No se encontraron diferencias estadísticamente significativas ni en el consumo de cocaína $\left(\chi^{2}(1)=\right.$ $1,57, p=0,209)$ ni en el de heroína $(\chi 2(1)=0,42, p=0,517)$.

\section{Asociación entre experiencias psicóticas atenuadas y consumo de sustancias}

A continuación, se analizó la relación entre las subescalas y las puntuaciones totales del PDI-21 y de la CAPE-42 y el consumo o no de sustancias. En la Tabla 1 se recogen las puntuaciones medias en función del consumo, así como del policonsumo global. Los resultados mostraron diferencias estadísticamente significativas en la mayor parte de las comparaciones llevadas a cabo. El valor $\lambda$ de Wilks reveló la presencia de diferencias estadísticamente significativas en función de los factores Alcohol $(\lambda=0,974, F(4,652)=4,289$, $p=0,002, \eta^{2}$ parcial $\left.=0,026\right)$, Cannabis $(\lambda=0,971, F(4,652)$ $=4,90, p=0,001, \eta^{2}$ parcial $\left.=0,029\right)$, Drogas de Diseño $(\lambda=$ $0,978, F(4,652)=3,735, p=0,005, \eta^{2}$ parcial $\left.=0,022\right)$ y Consumo total $\left(\lambda=0,969, F(4,652)=5,130, p<0,001, \eta^{2}\right.$ parcial $=0,031)$. No se hallaron diferencias estadísticamente significativas en función del factor Cocaína $(\lambda=0,961, F(4,652)$ $=4,010, p=0,348, \eta^{2}$ parcial $\left.=0,007\right)$. En todos los casos, el grupo de uso de sustancias presentó mayores puntuaciones medias totales en el PDI-21 y la CAPE-42 en comparación con el grupo de no consumidores.

Los resultados del análisis univariado de la varianza mostraron diferencias estadísticamente significativas en función del factor Alcohol en todas las puntuaciones, excepto en la dimensión depresiva de la CAPE-42 $(p<0,05)$. Atendiendo al patrón de consumo de alcohol (en función de los gr./día) se observaron diferencias estadísticamente significativas entre los diferentes grupos $(\lambda=0,933, F(20,2150)=2,284, p<0,001$, $\eta^{2}$ parcial $\left.=0,017\right)$. Concretamente, se encontraron diferencias estadísticamente significativas en la puntuación total del PDI-21 $\left(F(5,651)=5,946, p<0,001, \eta^{2}\right.$ parcial $\left.=0,044\right)$ y en su dimensión Positiva $\left(F(5,651)=4,604, p<0,001, \eta^{2}\right.$ parcial $=0,011)$, y en la puntuación total de la CAPE-42 $(F(5,651)=$ $3,899, p<0,001, \eta^{2}$ parcial $\left.=0,029\right)$. La tendencia hallada fue que los no bebedores presentaron puntaciones medias inferiores al resto de los grupos (excepto ex-bebedores o más de 50 gr./día), y que el grupo de consumo de 21-50 gr./día presentó puntuaciones medias superiores al resto de los grupos objeto de comparación. En el caso del consumo de cannabis y policonsumo total, los participantes que informaron positivamente de consumo presentaron también mayores puntuaciones medias en los citados autoinformes en comparación con el grupo de no uso de sustancias $(p<0,05)$. Por su parte, los participantes que presentaron consumo de drogas de diseño, solo presentaron puntuaciones medias estadísticamente su-

Tabla 1. Puntuaciones medias en el Inventario de Ideas Delirantes de Peters et al. 21 (PDI-21) y en la Escala para la Evaluación Comunitaria de las Experiencias Psíquicas-42 (CAPE-42) en función del consumo de sustancias

\begin{tabular}{|c|c|c|c|c|c|c|c|c|c|c|}
\hline & \multicolumn{2}{|l|}{$\begin{array}{l}\text { PDI-21 } \\
\text { Total }\end{array}$} & \multicolumn{2}{|c|}{$\begin{array}{l}\text { CAPE-42 } \\
\text { Positiva }\end{array}$} & \multicolumn{2}{|c|}{$\begin{array}{l}\text { CAPE-42 } \\
\text { Depresiva }\end{array}$} & \multicolumn{2}{|c|}{$\begin{array}{l}\text { CAPE-42 } \\
\text { Negativa }\end{array}$} & \multicolumn{2}{|c|}{$\begin{array}{l}\text { CAPE-42 } \\
\text { Total }\end{array}$} \\
\hline & Uso & No uso & Uso & No uso & Uso & No uso & Uso & No uso & Uso & No uso \\
\hline \multicolumn{11}{|l|}{ Alcohol } \\
\hline Media & 3,95 & 5,02 & 24,53 & 26,15 & 13,38 & 13,73 & 22,62 & 23,51 & 60,52 & 63,38 \\
\hline$D T$ & 2,60 & 3 & 3,56 & 5,76 & 2,67 & 2,98 & 4,57 & 4,93 & 8,69 & 10,56 \\
\hline \multicolumn{11}{|c|}{ Cannabis } \\
\hline Media & 4,04 & 5,32 & 24,71 & 26,42 & 13,42 & 13,78 & 22,69 & 23,75 & 60,82 & 63,95 \\
\hline$D T$ & 2,58 & 3,27 & 3,86 & 6,12 & 2,79 & 2,73 & 4,59 & 5,08 & 8,88 & 11,05 \\
\hline \multicolumn{11}{|c|}{ Cocaína } \\
\hline Media & 4,24 & 5,04 & 24,94 & 26,37 & 13,46 & 13,88 & 22,89 & 23,08 & 61,29 & 63,33 \\
\hline$D T$ & 2,81 & 2,24 & 4,38 & 5,11 & 2,81 & 2,41 & 4,75 & 4,14 & 9,48 & 8,73 \\
\hline \multicolumn{11}{|c|}{ Drogas de diseño } \\
\hline Media & 4,27 & 5,47 & 24,96 & 28,47 & 13,49 & 13,47 & 22,93 & 22,06 & 61,38 & 64 \\
\hline$D T$ & 2,77 & 2,85 & 4,35 & 6,78 & 2,79 & 2,29 & 4,72 & 4,1 & 9,43 & 9,51 \\
\hline \multicolumn{11}{|c|}{ Cualquier consumo } \\
\hline Media & 3,84 & 4,96 & 24,39 & 26,01 & 13,32 & 13,73 & 22,45 & 23,56 & 60,16 & 63,31 \\
\hline$D T$ & 2,56 & 2,95 & 3,44 & 5,47 & 2,72 & 2,85 & 4,59 & 4,8 & 8,67 & 10,16 \\
\hline
\end{tabular}

Nota. DT: Desviación típica; Alcohol: No consumo $n=445$, Consumo $n=215$; Cannabis: No consumo $n=527$, Consumo $n=133$; Cocaína: No consumo $n=609$, Consumo $n=51$; Drogas de diseño: No consumo $n=643$, Consumo $n=17$; Cualquier consumo: No consumo $n=389$, Consumo $n=217$. 
Tabla 2. Modelos de regresión lineal binaria de consumo de sustancias que predicen las puntuaciones extremas en el PDI-21 y en la CAPE-42, controlando el efecto del género y la ansiedad.

\begin{tabular}{|c|c|c|c|c|c|c|c|c|c|}
\hline Variable & $\begin{array}{l}\text { Tipo de } \\
\text { consumo }\end{array}$ & $B$ & $\begin{array}{l}\text { Error } \\
\text { estándar }\end{array}$ & Wald & g.l. & $p$ & $\begin{array}{c}\text { Odds } \\
\text { ratio }\end{array}$ & $\begin{array}{l}\text { Intervalo } \\
\text { confidencial } \\
95 \%\end{array}$ & $\begin{array}{l}\text { R2 } \\
\text { Nagelkerke }\end{array}$ \\
\hline \multicolumn{10}{|c|}{ PDI-21 Total } \\
\hline & Alcohol & 3,161 & 0,787 & 16,12 & 1 & 0,001 & 23,59 & $5,04-110,30$ & 0,557 \\
\hline & Cannabis & 1,434 & 0,677 & 9,48 & 1 & 0,034 & 4,195 & $1,112-15,22$ & 0,591 \\
\hline \multicolumn{10}{|c|}{ CAPE Positiva } \\
\hline & Alcohol & 0,875 & 0,444 & 3,875 & 1 & 0,049 & 2,399 & $1,004-5,371$ & 0,457 \\
\hline \multicolumn{10}{|c|}{ CAPE Depresiva } \\
\hline & Alcohol & 2,230 & 0,077 & 9,073 & 1 & 0,003 & 10,172 & $\begin{array}{l}2,248- \\
46,018\end{array}$ & 0,831 \\
\hline \multicolumn{10}{|c|}{ CAPE Total } \\
\hline & Alcohol & 1,572 & 0,747 & 4,429 & 1 & 0,035 & 4,816 & $\begin{array}{l}1,114- \\
20,822\end{array}$ & 0,796 \\
\hline
\end{tabular}

periores a los no consumidores en la dimensión Positiva de la CAPE-42 $\left(F(4,652)=7,183, p<0,001, \eta^{2}\right.$ parcial $\left.=0,011\right)$.

\section{Predicción de experiencias psicóticas atenuadas y consumo de sustancias}

Finalmente, se llevaron a cabo diferentes análisis de regresión logística binaria seleccionando a los participantes con puntuaciones extremas en el PDI-21 y la CAPE-42. Se controló el efecto del género, la ansiedad estado y la ansiedad rasgo. Los resultados de los análisis de regresión logística se presentan en la Tabla 2. Como se puede observar, el consumo de alcohol fue la variable que resultó ser estadísticamente significativa en la mayoría de los modelos estimados. En el caso del consumo de cannabis, éste predijo únicamente la puntuación total de los participantes con puntuaciones extremas en el PDI-21. Ninguno de los diferentes tipos de consumo de sustancias predijo de forma estadísticamente significativa las puntuaciones en la dimensión Negativa de la CAPE-42. La cocaína y las drogas de diseño no predijeron las puntuaciones extremas, ni del PDI-21 ni de la CAPE-42.

\section{Discusión y conclusiones}

El principal objetivo del presente estudio fue examinar la relación entre las experiencias psicóticas atenuadas y el consumo de sustancias en una muestra de universitarios. Los resultados mostraron que un $96 \%$ de la muestra informó de alguna experiencia de ideación delirante, mientras que el 20,3\% informó de, al menos, una experiencia psicotiforme de tipo positivo. Por su parte, el 41,1\% de la muestra refirió algún consumo de sustancias. Los participantes consumidores de cualquier sustancia, excepto cocaína, informaron de un mayor número de experiencias psicóticas subclínicas, sobre todo de tipo cognitivo-perceptual. Asimismo, el consumo de alcohol predijo, en la mayoría de los casos, puntuaciones extremas en las medidas de ideación delirante (PDI-21) y experiencias psicóticas atenuadas (CAPE-42). Estos hallazgos parecen apoyar los modelos dimensionales del fenotipo psicótico y permiten mejorar la comprensión de la relación entre las experiencias pseudo-psicóticas y el consumo de sustancias en jóvenes adultos no clínicos.

En primer lugar, los hallazgos encontrados en este trabajo indican que las experiencias psicóticas no se circunscriben únicamente a la población clínica, sino que se pueden encontrar distribuidas normalmente entre la población general, por debajo del umbral clínico, sugiriendo la posibilidad de una continuidad entre los fenotipos clínico y subclínico de la psicosis (Linscott y van Os, 2013; van Os et al., 2009). Estudios previos han llegado a conclusiones similares, analizando tanto adolescentes como adultos no clínicos de la población general (Debbané et al., 2015; Fonseca-Pedrero, Santarén-Rosell, Paino, y Lemos Giraldez, 2013; Ibáñez-Casas et al., 2015; McGrath et al., 2015). Por ejemplo, en un estudio llevado a cabo por McGrath et al. (2015), utilizando una muestra de 31.261 adultos procedentes de 18 países, se encontró que la prevalencia media de haber tenido una experiencias psicótica atenuada era del $5,8 \%$, siendo para las experiencias alucinatorias del $5,2 \%$ y del $1,3 \%$ para las experiencias delirantes. Asimismo, el consumo de sustancias fue bastante común en esta muestra, siendo los resultados similares a los informados en trabajos previos o incluso inferiores (Fumero et al., 2009; Hernández-Serrano, Font-Mayolas, y Gras, 2015). Según la Encuesta Domiciliaria sobre Alcohol y otras Drogas 2013/2014 (EDADES, 2015), las drogas de mayor consumo son el alcohol (el 78,3\% lo ha consumido en los últimos doce meses), el tabaco $(40,7 \%)$ y los hipnosedantes (12,2\%). Asimismo, en cuanto al consumo de cannabis, el 9,2\% de los encuestados indicó que lo había probado 
alguna vez en el último año, mientras que el 6,6\% alguna vez en el último mes; concretamente, el 2,2\% de la población española cumple los criterios requeridos para el diagnóstico de consumo problemático de cannabis.

En segundo lugar, los participantes que consumían alguna sustancia, excepto cocaína, informaron de un mayor número de experiencias psicóticas atenuadas. Concretamente, los tamaños del efecto fueron más altos para los consumos de alcohol y de cannabis. Los resultados encontrados en el presente trabajo son convergentes con los informados en la literatura previa, tanto en el campo de las experiencias psicóticas atenuadas (Barkus et al., 2006; Mackie et al., 2013; Saha et al., 2011; Schubart et al., 2011; van Os et al., 2002) como en el de la esquizotipia (Barkus et al., 2006; Davis et al., 2013; Fumero et al., 2009; Najolia et al., 2012; Nunn et al., 2001). Por ejemplo, Saha et al. (2011) hallaron que las experiencias delirantes atenuadas se relacionaban con consumo de tabaco, cannabis y alcohol. Asimismo, los participantes con trastornos por consumo de cannabis fueron más propensos a informar de experiencias subclínicas psicóticas. Esta asociación era más prominente en aquellos individuos con un inicio de consumo en los 16 años o anterior. En contraste, el patrón de asociación entre experiencias delirantes y el uso o dependencia del alcohol fue menos consistente. No obstante, las personas con trastornos por consumo de alcohol de aparición temprana eran más propensas a informar de este tipo de experiencias atenuadas (Saha et al., 2011).

Atendiendo a estos resultados, los participantes que consumen sustancias refieren más experiencias pseudo-psicóticas en comparación con aquellos que no consumen, si bien la naturaleza de tal asociación aún no está clara y parece ser bastante compleja. Se podrían barajar diferentes hipótesis. Es posible que los participantes con este tipo de experiencias psicóticas recurran a sustancias para "mitigar" o "aliviar" su impacto (p.ej., disforia, automedicación o causalidad inversa). De igual forma, resulta plausible que el propio consumo de drogas predisponga a las experiencias psicóticas (relación causal). También es posible que exista una relación bidireccional (una combinación de ambas hipótesis) o que simplemente se encuentren asociadas. En este sentido, los modelos actuales consideran que las relaciones entre consumo de sustancias y la experiencias, los síntomas y los trastornos psicóticos son complejas, dinámicas y multifactoriales (p.ej., edad de inicio, frecuencia de consumo, factores protectores, historia familiar de trastorno), siendo las vías para el desarrollo de un cuadro psicótico heterogéneas (Henquet, Di Forti, Morrison, Kuepper, y Murray, 2008).

En tercer lugar, los resultados derivados del análisis de regresión logística mostraron que la variable con más poder de predicción en el grupo con puntuaciones extremas en experiencias psicóticas atenuadas, una vez controlado el efecto de determinadas covariables, fue el consumo de alcohol. En casi todos los casos las odds ratio encontradas fueron elevadas. Los consumidores de alcohol con un uso moderado-alto (21-50 gr./día) fueron los que mayores puntuaciones medias obtuvieron en comparación con el resto de patrones. No se pueden extraer datos concluyentes del grupo de consumo grave (más de 50 gr./día) dado que el tamaño muestral en este estudio fue reducido. Del mismo modo, la asociación entre el tipo de consumo y las experiencias psicotiformes mostró un patrón diferenciado, encontrándose una relación más fuerte con las experiencias psicóticas de tipo cognitivo-perceptual y menos con las negativas. Trabajos previos que analizan el papel de los rasgos negativos de la esquizotipia o las experiencias psicóticas negativas de tipo atenuado, encuentran resultados similares aunque inconsistentes. Por ejemplo, algunos estudios reflejan la existencia de asociación entre la dimensión negativa y el consumo a nivel subclínico (Bailey y Swallow, 2004; Davis et al., 2013); no obstante, es igualmente cierto que otros estudios hallan que los consumidores de cannabis y alcohol presentan menores puntuaciones en la dimensión negativa en comparación con los no consumidores (Nunn et al., 2001; Skosnik et al., 2008) o no encuentran tal asociación (Barkus et al., 2006; Dumas et al., 2002; Fridberg et al., 2011; Schiffman et al., 2005). En términos generales, estos resultados parecen indicar que existe un patrón de asociación diferenciado entre este conjunto de experiencias y rasgos psicóticos subclínicos y el consumo de sustancias (p. ej., uso, frecuencia y edad de inicio), si bien se debe seguir profundizando en esta cuestión, ya que los resultados varían en función del tipo de estudio, muestra, instrumento de medida y análisis estadístico empleado.

Este tipo de experiencias psicóticas atenuadas se podría utilizar en combinación con otros marcadores de riesgo, como por ejemplo, el consumo o abuso de sustancias, estrés, trauma o urbanicidad (van Os et al., 2010), con la finalidad de examinar posibles mecanismos etiológicos subyacentes a los cuadros del espectro psicótico (Henquet et al., 2008). Algunos modelos teóricos hipotetizan que la presencia aditiva o sinérgica de diferentes factores de riesgo medioambientales, dependiendo del periodo del desarrollo del individuo y de la predisposición o vulnerabilidad, pueden favorecer que determinadas experiencias psicóticas atenuadas se vuelvan anormalmente persistentes y acaben desarrollando, en un futuro, un cuadro clínico y la consiguiente necesidad de tratamiento (Cougnard et al., 2007). Por ejemplo, estudios epidemiológicos han encontrado que la iniciación en el uso de cannabis en la adolescencia incrementa la probabilidad de síntomas y trastornos psicóticos en la edad adulta (Arseneault et al., 2002) y favorece su persistencia en el tiempo (Mackie et al., 2013). Del mismo modo, parece ser que si el consumo de cannabis interactúa con otros factores de riesgo como son las experiencias traumáticas, aumenta la probabilidad de presencia de síntomas psicóticos (Cougnard et al., 2007; Harley et al., 2010). Otros modelos, basados en estudios animales, también postulan sobre el efecto que los cannabinoides puedan ocasionar en diferentes receptores dopaminérgicos y/o cannabinoides (especialmente a nivel 
del córtex prefrontal, estriado e hipocampo), así como acerca de su capacidad de generar modificaciones en el sistema glutamatérgico e inducir una cascada de cambios bioquímicos, tal vez neurotóxicos, y de igual forma, incrementando el riesgo de desarrollar trastornos psicóticos (Bossong y Niesink, 2010; Rocchetti et al., 2013).

Los resultados encontrados en el presente estudio deberían interpretarse a la luz de las siguientes limitaciones. En primer lugar, se ha utilizado una muestra de conveniencia de estudiantes universitarios, predominantemente mujeres. En este sentido, las características de la muestra afectan a la validez de los resultados encontrados, así como a la posible generalización de los mismos a otras poblaciones de interés. En segundo lugar, existe la problemática inherente a la aplicación de cualquier tipo de autoinforme, por lo que hubiese sido interesante la utilización de informantes externos (p.ej., entrevistas) para analizar la presencia de trastornos mentales entre los participantes del estudio, o medidas de laboratorio (p.ej., analíticas de sangre) para verificar el consumo de sustancias. En tercer lugar, no se debe perder de vista la naturaleza transversal de esta investigación, con lo que no es posible establecer inferencias de causa-efecto. En cuarto lugar, el consumo de sustancias se ha asociado a déficits neurocognitivos (López-Caneda et al., 2014) y efectos fisiológicos (Vinader-Caerols, Monleon, y Parra, 2014), aspectos que pueden modular los resultados encontrados. Finalmente, hubiese sido interesante la administración de instrumentos de medida que analizaran el estado afectivo de tipo depresivo.

Futuros estudios deberían llevar a cabo estudios longitudinales y determinar el valor predictivo del consumo de sustancias en el desarrollo de trastornos del espectro psicótico, así como incorporar modelos neurocientíficos que tengan en cuenta las complejas y dinámicas interacciones que se establecen en el interfaz gen x ambiente; todo ello con la finalidad de mejorar las estrategias de detección precoz, así como de identificación y prevención temprana en personas de riesgo a los trastornos del espectro psicótico.

\section{Agradecimientos}

Esta investigación ha sido financiada por el Ministerio de Ciencia e Innovación de España (MICINN), por el Instituto Carlos III, Centro de Investigación Biomédica en Red de Salud Mental (CIBERSAM) y por la Convocatoria 2015 de Ayudas Fundación BBVA a Investigadores y Creadores Culturales. Referencias de los proyectos: PSI2011-28638, PSI 2011-23818 y PSI2014-56114-P.

\section{Referencias}

Addington, J., Case, N., Saleem, M. M., Auther, A. M., Cornblatt, B. A., y Cadenhead, K. S. (2014). Substance use in clinical high risk for psychosis: a review of the literature. Early Intervention in Psychiatry, 8, 104-112.
Arseneault, L., Cannon, M., Poulton, R., Murray, R., Caspi, A., y Moffitt, T. E. (2002). Cannabis use in adolescence and risk for adult psychosis: longitudinal prospective study. British Medical Journal, 325, 1212-1213.

Bailey, E. L., y Swallow, B. L. (2004). The relationship between cannabis use and schizotypal symptoms. European Psychiatry, 19, 113-114.

Barkus, E. J., Stirling, J., Hopkins, R. S., y Lewis, S. (2006). Cannabis-induced psychosis-like experiences are associated with high schizotypy. Psychopathology, 39, 175-178.

Barragan, M., Laurens, K. R., Blas Navarro, J., y Obiols, J. E. (2011). 'Theory of Mind', psychotic-like experiences and psychometric schizotypy in adolescents from the general population. Psychiatry Research, 186, 225-131.

Bossong, M. G., y Niesink, R. J. (2010). Adolescent brain maturation, the endogenous cannabinoid system and the neurobiology of cannabis-induced schizophrenia. Progress in Neurobiology, 92, 370-385.

Broussard, B., Kelley, M. E., Wan, C. R., Cristofaro, S. L., Crisafio, A., Haggard, P. J., ... Compton, M. T. (2013). Demographic, socio-environmental, and substance-related predictors of duration of untreated psychosis (DUP). Schizophrenia Research, 148, 93-98.

Buckley, P. F., Miller, B. J., Lehrer, D. S., y Castle, D. J. (2009). Psychiatric comorbidities and schizophrenia. Schizophrenia Bulletin, 35, 383-402.

Casadio, P., Fernandes, C., Murray, R. M., y Di Forti, M. (2011). Cannabis use in young people: the risk for schizophrenia. Neuroscience E Biobehavioral Reviews, 35, 17791787.

Cougnard, A., Marcelis, M., Myin-Germeys, I., De Graaf, R., Vollebergh, W., Krabbendam, L., ... Van Os, J. (2007). Does normal developmental expression of psychosis combine with environmental risk to cause persistence of psychosis? A psychosis proneness-persistence model. Psychological Medicine, 37, 513-527.

Davis, G. P., Compton, M. T., Wang, S., Levin, F. R., y Blanco, C. (2013). Association between cannabis use, psychosis, and schizotypal personality disorder: findings from the National Epidemiologic Survey on Alcohol and Related Conditions. Schizophrenia Research, 151, 197-202.

Debbané, M., Eliez, S., Badoud, D., Conus, P., Flückiger, R., y Schultze-Lutter, F. (2015). Developing psychosis and its risk states through the lens of schizotypy. Schizophrenia Bulletin, 41, S396-407.

Dumas, P., Saoud, M., Bouafia, S., Gutknecht, C., Ecochard, R., Daléry, J., ... d'Amato, T. (2002). Cannabis use correlates with schizotypal personality traits in healthy students. Psychiatry Research, 109, 27-35.

EDADES. (2015). Encuesta Domiciliaria sobre Alcohol y otras Drogas 2013/2014. Madrid.: Ministerio de Sanidad, Servicios Sociales e Igualdad Secretaría de Estado de Servicios Sociales e Igualdad. Delegación del Gobierno para el Plan Nacional sobre Drogas. 
Fonseca-Pedrero, E., Lemos-Giráldez, S., Paino, M., Santarén-Rosell, M., Sierra-Baigrie, S., y Ordóñez-Camblor, N. (2011). Instrumentos de medida para la evaluación del fenotipo psicótico [Measuring instruments for evaluation phenotype of psychotic]. Papeles del Psicólogo, 32, 129-151.

Fonseca-Pedrero, E., Paino, M., y Fraguas, D. (2013). DSM5: ¿Síndrome de psicosis atenuada? Papeles del Psicólogo, 34, 190-207.

Fonseca-Pedrero, E., Paino, M., Lemos-Giráldez, S., y Muñiz, J. (2012a). Propiedades psicométricas del Cuestionario de Ansiedad Estado Rasgo en universitarios. Psicología Conductual, 20, 547- 561.

Fonseca-Pedrero, E., Paino, M., Lemos-Giráldez, S., y Muñiz, J. (2012b). Validation of the Community Assessment Psychic Experiences-42 (CAPE-42) in Spanish college students and patients with psychosis. Actas Españolas de Psiquiatría, 40, 169-176.

Fonseca-Pedrero, E., Paino, M., Santarén-Rosell, M., Lemos-Giráldez, S., y Muñiz, J. (2012). Psychometric properties of the Peters et al Delusions Inventory 21 in college students. Comprehensive Psychiatry, 53, 893-899.

Fonseca-Pedrero, E., Santarén-Rosell, M., Paino, M., y Lemos Giraldez, S. (2013). Cluster A maladaptive personality patterns in a non-clinical adolescent population. Psicothema, 25, 171-178.

Fridberg, D. J., Vollmer, J. M., O’Donnell, B. F., y Skosnik, P. D. (2011). Cannabis users differ from non-users on measures of personality and schizotypy. Psychiatry Research, 186, 46-52.

Fumero, A., Santamaría, C., y Navarrete, G. (2009). [Predisposition to alcohol and drug consumption in schizophrenia-vulnerable people]. Revista de Neurología, 49, 8-12.

González-Pinto, A., Alberich, S., Barbeito, S., Gutierrez, M., Vega, P., Ibáñez, B., ... Arango, C. (2011). Cannabis and first-episode psychosis: different long-term outcomes depending on continued or discontinued use. Schizophrenia Bulletin, 37, 631-639.

Guillén-Riquelme, A., y Buela-Casal, G. (2011). Actualización psicométrica y funcionamiento diferencial de los ítems en el State Trait Anxiety Inventory (STAI). Psicothema, 23, 510-515.

Harley, M., Kelleher, I., Clarke, M., Lynch, F., Arseneault, L., Connor, D., ... Cannon, M. (2010). Cannabis use and childhood trauma interact additively to increase the risk of psychotic symptoms in adolescence. Psychological Medicine, 40, 1627-1634.

Henquet, C., Di Forti, M., Morrison, P., Kuepper, R., y Murray, R. M. (2008). Gene-environment interplay between cannabis and psychosis. Schizophrenia Bulletin, 34, 1111-1121.

Henquet, C., Krabbendam, L., Spauwen, J., Kaplan, C., Lieb, R., Wittchen, H. U., y van Os, J. (2005). Prospective cohort study of cannabis use, predisposition for psychosis, and psychotic symptoms in young people. British Medical Journal 330, 11-14.
Henquet, C., Murray, R., Linszen, D., y Van Os, J. (2005). The environment and schizophrenia: The role of cannabis use. Schizophrenia Bulletin, 31, 608-612.

Henquet, C., van Os, J., Kuepper R., Delespaul, P., Smits, M., Campo, J. A., y Myin-Germeys, I. (2010). Psychosis reactivity to cannabis use in daily life: an experience sampling study. British Journal of Psychiatry, 196, 447-453.

Hernández-Serrano, O., Font-Mayolas, S., y Gras, M. E. (2015). Policonsumo de drogas y su relación con el contexto familiar y social en jóvenes universitarios. Adicciones, 27, 205-213.

Ibáñez-Casas, I., Femia-Marzo, P., Padilla, J. L., Green, C. E., de Portugal, E., y Cervilla, J. A. (2015). Spanish adaptation of the Green Paranoid Thought Scales. Psicothe$m a, 27,74-81$.

Kuepper, R., van Os, J., Lieb, R., Wittchen, H. U., Höfler, M., y Henquet, C. (2011). Continued cannabis use and risk of incidence and persistence of psychotic symptoms: 10 year follow-up cohort study. British Medical Journal, 342, d738.

Kwapil, T. R. (1996). A longitudinal study of drug and alcohol use by psychosis-prone and impulsive-nonconforming individuals. Journal of Abnormal Psychology, 105, 114-123.

Kwapil, T. R., y Barrantes-Vidal, N. (2015). Schizotypy: Looking back and moving forward. Schizophrenia Bulletin, 41, S366-373.

Lambert, M., Conus, P., Lubman, D. I., Wade, D., Yuen, H., Moritz, S., ... Schimmelmann, B. G. (2005). The impact of substance use disorders on clinical outcome in 643 patients with first-episode psychosis. Acta Psychiatrica Scandinavica, 112, 141-148.

Linscott, R. J., y van Os, J. (2013). An updated and conservative systematic review and meta-analysis of epidemiological evidence on psychotic experiences in children and adults: on the pathway from proneness to persistence to dimensional expression across mental disorders. Psychological Medicine, 43, 1133-1149.

López-Caneda, E., Mota, N., Crego, A., Velásquez, T., Corral, M., Rodríguez Holguín, S., y Cadaveira, F. (2014). Anomalías neurocognitivas asociadas al consumo intensivo de alcohol (binge drinking) en jóvenes y adolescentes: Una revisión. Adicciones, 26, 334-359.

López-Ilundain, J. M., Pérez-Nievas, F., Otero, M., y Mata, I. (2006). Inventario de experiencias delirantes de Peters (PDI) en pblación general española: fiabilidad interna, estructura factorial y asociación con variables demográficas. Actas Españolas de Psiquiatría, 34, 94-104.

Mackie, C. J., O’Leary-Barrett, M., Al-Khudhairy, N., Castellanos-Ryan, N., Struve, M., Topper, L., y Conrod, P. (2013). Adolescent bullying, cannabis use and emerging psychotic experiences: a longitudinal general population study. Psychological Medicine, 43, 1033-1044.

McGrath, J., Welham, J., Scott, J., Varghese, D., Degenhardt, L., Hayatbakhsh, M. R., ... Najman, J. M. (2010). Association between cannabis use and psychosis-related 
outcomes using sibling pair analysis in a cohort of young adults. Archives of General Psychiatry, 67, 440-447.

McGrath, J. J., Saha, S., Al-Hamzawi, A., Alonso, J., Bromet, E. J., Bruffaerts, R., ... Kessler, R. C. (2015). Psychotic Experiences in the General Population: A Cross-National Analysis Based on 31,261 Respondents From 18 Countries. JAMA Psychiatry, 72, 697-705.

McGuire, P. K., Jones, P., Harvey, I., Williams, M., McGuffin, P., y Murray, R. M. (1995). Morbid risk of schizophrenia for relatives of patients with cannabis-associated psychosis. Schizophrenia Research, 15, 277-281.

Moore, T. H., Zammit, S., Lingford-Hughes, A., Barnes, T. R., Jones, P. B., Burke, M., y Lewis, G. (2007). Cannabis use and risk of psychotic or affective mental health outcomes: a systematic review. Lancet, 370, 319-328.

Muñiz, J., Elosua, P., y Hambleton, R. K. (2013). Directrices para la traducción y adaptación de los tests: segunda edición [International Test Commission Guidelines for test translation and adaptation: Second edition]. Psicothema, 25, 151-157.

Najolia, G. M., Buckner, J. D., y Cohen, A. S. (2012). Cannabis use and schizotypy: the role of social anxiety and other negative affective states. Psychiatry Research, 200, 660-668.

Nunn, J. A., Rizza, F., y Peters, E. R. (2001). The incidence of schizotypy among cannabis and alcohol users. Journal of Nervous and Mental Disease, 189, 741-748.

Peters, E., Joseph, S., Day, S., y Garety, P. A. (2004). Measuring Delusional Ideation: The 21- Item Peters et al. Delusion Inventory. Schizophrenia Bulletin, 30, 1005-1022.

Rocchetti, M., Crescini, A., Borgwardt, S., Caverzasi, E., Politi, P., Atakan, Z., y Fusar-Poli, P. (2013). Is cannabis neurotoxic for the healthy brain? A meta-analytical review of structural brain alterations in non-psychotic users. Psychiatry and Clinical Neurosciences, 67, 483-492.

Saha, S., Scott, J. G., Varghese, D., Degenhardt, L., Slade, T., y McGrath, J. J. (2011). The association between delusional-like experiences, and tobacco, alcohol or cannabis use: a nationwide population-based survey. BMC Psychiatry, 11, 202.

Schiffman, J., Nakamura, B., Earleywine, M., y LaBrie, J. (2005). Symptoms of schizotypy precede cannabis use. Psychiatry Research, 134, 37-42.

Schubart, C. D., van Gastel, W. A., Breetvelt, E. J., Beetz, S. L., Ophoff, R. A., Sommer, I. E., ... Boks, M. P. (2011). Cannabis use at a young age is associated with psychotic experiences. Psychological Medicine, 41, 1301-1310.

Skosnik, P. D., Park, S., Dobbs, L., y Gardner, W. L. (2008). Affect processing and positive syndrome schizotypy in cannabis users. Psychiatry Research, 157, 279-282.

Soto-Brandt, G., Portilla Huidobro, R., Huepe Artigas, D., Rivera-Rei, Á., Escobar, M. J., Salas Guzmán, N., ... Castillo-Carniglia, Á. (2014). Evidencia de validez en Chile del Alcohol, Smoking and Substance Involvement Screening Test (ASSIST). Adicciones, 26, 291-302.
Spielberger, C. D., Gorsuch, R., Lushene, R., Vagg, P. R., y Jacobs, G. A. (1983). Manual for the State-Trait Anxiety Inventory (STAI). Palo Alto, CA: Consulting Psychologists Press.

Spielberger, C. D., Gorsuch, R. L., y Lushene, R. E. (2008). STAI. Cuestionario de Ansiedad Estado-Rasgo ( $7^{a}$ ed. revisada). Madrid: TEA Ediciones S.A.

Statistical Package for the Social Sciences. (2006). SPSS Base 15.0 User's Guide. Chicago, IL: SPSS Inc.

Stefanis, N. C., Dragovic, M., Power, B. D., Jablensky, A., Castle, D., y Morgan, V. A. (2014). The effect of drug use on the age at onset of psychotic disorders in an Australian cohort. Schizophrenia Research, 156, 211-216

Stefanis, N. C., Hanssen, M., Smirnis, N. K., Avramopoulos, D. A., Evdokimidis, I. K., Stefanis, C. N., ... Van Os, J. (2002). Evidence that three dimensions of psychosis have a distribution in the general population. Psychological Medicine, 32, 347-358.

Stowkowy, J., y Addington, J. (2013). Predictors of a clinical high risk status among individuals with a family history of psychosis. Schizophrenia Research, 147, 281-286.

Valmaggia, L. R., Day, F. L., Jones, C., Bissoli, S., Pugh, C., Hall, D., ... McGuire, P. K. (2014). Cannabis use and transition to psychosis in people at ultra-high risk. Psychological Medicine, 44, 2503-2512.

van Os, J., Bak, M., Hanssen, M., Bijl, R. V., de Graaf, R., y Verdoux, H. (2002). Cannabis use and psychosis: a longitudinal population-based study. American Journal of Epidemiology, 156, 319-327.

van Os, J., Kenis, G., y Rutten, B. P. (2010). The environment and schizophrenia. Nature, 468, 203-312.

van Os, J., Linscott, R. J., Myin-Germeys, I., Delespaul, P., y Krabbendam, L. (2009). A systematic review and meta-analysis of the psychosis continuum: Evidence for a psychosis proneness-persistence-impairment model of psychotic disorder. Psychological Medicine, 39, 179-195.

Vinader-Caerols, C., Monleon, S., y Parra, A. (2014). Physiological and psychological effects of a high dose of alcohol in young men and women. Adicciones, 26, 238-246.

Wade, D., Harrigan, S., Edwards, J., Burgess, P. M., Whelan, G., y McGorry, P. D. (2006). Substance misuse in first-episode psychosis: 15-month prospective follow-up study. British Journal of Psychiatry, 189, 229-234.

Wisdom, J. P., Manuel, J. I., y Drake, R. E. (2011). Substance use disorder among people with first-episode psychosis: a systematic review of course and treatment. Psychiatric Services, 62, 1007-1012.

Yung, A. R., Yuen, H. P., Berger, G., Francey, S., Hung, T. C., Nelson, B., ... McGorry, P. (2007). Declining transition rate in ultra high risk (prodromal) services: dilution or reduction of risk? Schizophrenia Bulletin, 33, 673-681.

Zammit, S., Moore, T. H., Lingford-Hughes, A., Barnes, T. R., Jones, P. B., Burke, M., y Lewis, G. (2008 ). Effects of cannabis use on outcomes of psychotic disorders: systematic review. British Journal of Psychiatry, 193, 357-363. 